\title{
Desplazamiento de los docentes de lengua castellana a otras prácticas profesionales
}

\author{
Ana Lucía Cañón Moreno ${ }^{1}$
}

Recibido: 24-07-2018

Aceptado: 25-09-2018

\section{RESUMEN}

Este artículo de reflexión pretendió realizar una exploración teórica y docta sobre el hecho del desplazamiento de la formación pedagógica de los docentes de lengua castellana a énfasis diversos. Esto se logró a través de la búsqueda en fuentes secundarias que plantearan hipótesis que explicaran el hecho de la formación de docentes de lengua castellana habilitados para ejercer en otros campos profesionales relacionados con el lenguaje y de la entrevista a tres expertos en la formación de docentes de lengua castellana en América Latina (Venezuela, México y Colombia). El tema central abordó las posibles causas de la pérdida de primacía del asunto pedagógico en la formación de docentes de lengua castellana y las implicaciones socio - formativas de este hecho. Algunas de las conclusiones que explican este fenómeno se refieren a la pregnancia de las nuevas tecnologías (Konieczny, 2015), a la explosión del gusto por la literatura y la escritura y a las industrias culturales.

Palabras clave: formación docente, desplazamiento pedagógico, nuevos campos profesionales. 


\title{
Displacement of teachers of Castilian language to other professional practices
}

\begin{abstract}
This reflection article sought to make a learned and theoretical exploration on the fact of the displacement of the pedagogical training of teachers of Spanish language to different emphasis. This is accomplished through the search on secondary sources raised hypothesis explaining the fact enabled Spanish language teacher training to practice in other professional fields associated with the language and the interview with three experts in the training of teachers of Spanish language in Latin America (Venezuela, Mexico and Colombia). The central theme dealt with the causes of the loss of primacy of the pedagogical topic in the training of teachers of Spanish language and the implications of socio - educational of this fact. Some of the conclusions that explain this phenomenon refers to the weight of the new technologies, cultural industries and the explosion of taste in literature and writing.
\end{abstract}

Keywords: teacher training, pedagogical shift, new professional fields.

\section{Introducción - presentación}

En el año 2015, a partir de la Convocatoria de Investigación No. 10, del Centro de Investigación de la Universidad Santo Tomás, se llevó a cabo la investigación Tendencias en la formación actual de docentes de Lengua Castellana en América Latina, con el propósito de identificar las tendencias y de reconocer el énfasis de los diferentes planes de estudio de los programas de formación de docentes de Lengua Castellana en Latinoamérica a partir de la revisión de los mismos y sondear el campo de acción de los egresados de los programas consultados.

Por ello mismo, la investigación arrojó: 
Perfiles de ingreso y egreso de las universidades latinoamericanas consultadas; así como los posibles campos de acción de los egresados de tales programas, que incluyen docencia, investigación en lengua y literatura, producción editorial, gestión y gerencia cultural, producción de textos y traducción (Cañón y Cedeño, 2015,p. ).

El ejercicio investigativo motivó el reconocimiento de 37 planes de estudio, de 44 universidades consultadas. Se llegó a los resultados a través de la recolección y el análisis de la documentación revisada, lo que permitió determinar cuál es el enfoque de formación académica de los docentes de lengua castellana en tales universidades, encontrándose que sus énfasis se ofrecen en los siguientes campos del conocimiento: la educación, la pedagogía, la gestión y la gerencia de la cultura, la lingüística, la literatura, el trabajo editorial, el desarrollo histórico de la lengua y la investigación en estas mismas áreas.

Los perfiles de egreso muestran:

La importancia de una visión multidisciplinar que permite la comprensión - desde lo humano - del mundo contemporáneo; la preparación sólida que favorezca adelantar estudios de posgrado; el ingreso a la docencia y la posibilidad de desarrollar investigaciones; el desenvolvimiento profesional en los diferentes medios de comunicación; la competencia para el trabajo editorial; amplias capacidades de lectura y escritura; sensibilidad frente a los problemas sociales; conocimiento profundo de la lengua española y su funcionamiento. (Cañón y Cedeño, 2015, p. ).

La investigación arriba citada evidenció que los egresados de los programas ya mencionados, podrían desempeñarse como docentes, investigadores, creadores literarios, comunicadores sociales, promotores y administradores de industrias culturales.La promoción cultural se evidencia en la posibilidad de trabajo en la gerencia de Casas de Cultura, guionistas de radio, cine y televisión, la asesoría de bibliotecas, la elaboración de prólogos y la reseña de libros, la traducción de textos.

El presente artículo pretende indagar, de manera preliminar, cuáles son las causas e implicaciones de una mayor formación disciplinar en los estudiantes de licenciatura en lengua castellana, frente a un menor 
énfasis en los campos de la pedagogía y la didáctica, centrales en la labor docente.

\section{Metodología}

Dado el carácter exploratorio de la reflexión que se plantea en este artículo, el método con el que se adelantó, consistió en la realización de tres entrevistas semiestructuradas al mismo número de expertos, en temas relacionados con el lenguaje, la lengua, la pedagogía y la formación de docentes de lengua castellana; en la revisión de algunas fuentes secundarias que plantean hipótesis que explican, en alguna medida, el asunto de la formación de docentes de lengua castellana habilitados para ejercer en campos diferentes de la docencia.

Las preguntas base de la entrevista a los expertos fueron:

¿A qué atribuye el fenómeno de que la formación de docentes de lengua castellana en América Latina se esté orientando (en algunos casos) a perfiles ocupacionales diversos?

¿Qué fortalezas y qué debilidades considera que se desprenden del hecho mencionado anteriormente en la formación de docentes de lengua castellana?

¿Qué acciones se tendrían que adelantar para que la formación de los docentes le concediera la misma importancia al asunto pedagógico que al disciplinar?

\section{Revisión de la literatura (marco conceptual)}

A pesar de alguna buena intención del Estado colombiano, para profesionalizar la formación docente a través del Decreto 0272 de Febrero 11 de 1998, que establece los requisitos de creación y funcionamiento de los programas académicos de pregrado y postgrado en Educación ofrecidos por las universidades y por las instituciones universitarias de Colombia, decreto que exigió a las Facultades de Educación, hace veinte 
años, reformular sus concepciones y metodologías situando la pedagogía en el centro del currículo, sigue teniendo lo pedagógico muy poco peso en la formación de maestros (Viejo, Cabezas \& Martínez, 2013). Pareciera que continúa siendo más atractiva la idea de ser físico o biólogo o lingüista que la de ser maestro de las áreas de física, biología o lenguaje.

Los programas académicos en Educación corresponden a un campo de acción cuya disciplina fundante es la pedagogía, incluyendo en ella la didáctica, por cuanto constituye un ámbito de reflexión a partir del cual se genera conocimiento propio que se articula interdisciplinariamente". (art.2)

Realmente no se advierten fortalezas. Antes de 1998, cuando los currículos no aspiraban a que la pedagogía fuera "la disciplina fundante", como lo estableció el decreto ya mencionado, del 11 de febrero de tal año, la formación de licenciados en lingüística era extraordinariamente sólida en lo disciplinar y preocupantemente frágil en lo profesional.

Los programas de formación en el campo del lenguaje - existentes en el momento - atendían con mucha fuerza los pormenores teóricos involucrados en su objeto de estudio. Allí había una fortaleza indiscutible, así como era indiscutible también la debilidad pedagógica (de la Calle et al., 2014). Con los nuevos programas lo que era fuerte se perdió y lo que era débil continuó siendo débil. Hoy, hay que señalarlo, se están formando profesionales de baja talla con debilidades diagnosticadas en los dos frentes de formación: lo disciplinar y lo profesional pedagógico (Langer, 2016). Así que tanto los intereses de los futuros profesionales de la educación como el ideario de quienes construyen, administran y ejecutan las estructuras curriculares que forman maestros en Lengua castellana, oscilan entre esos dos extremos: por una parte, el apego fuerte a lo teórico - disciplinar - por riguroso, desdeñándose lo pedagógico, por sentirse este, un saber cotidiano y simple, y por otra, el interés fuerte por lo literario, por su gracia y novedad placentera. Interés que coexiste con cierta desatención por la enseñanza y el aprendizaje, asuntos sentidos por ellos como poco válidos (Acevedo, 2014).

La escuela tiene la misión fundamental de promover el desarrollo de la cognición humana (Aparicio, 2018).Si las facultades cognoscitivas son desarrollables debe contestarse entonces la pregunta de quién debe responsabilizarse de patrocinar su despliegue. Para ese interrogante existen tentativamente las siguientes respuestas. a) el medio social en 
general mediante una actividad espontánea; b) el núcleo familiar, c) un profesional independiente, especializado diferente del maestro y d) la escuela misma (Moreno, 1998)

Obviamente, la responsabilidad mayor estaría en la escuela, pues para ello fue creada y, por lo tanto, la formación de los docentes debe apuntarle a su desarrollo tanto en los aspectos disciplinares como en los pedagógicos, porque de otra manera no podrá contribuir con lo arriba planteado (Ferreyra, 2014).

Siguiendo con Moreno (1998), él insiste en "la necesidad de superar los paradigmas pedagógicos actuales caracterizados por el apego a las jerarquías, el predominio de la verbalidad, la tendencia reproductiva, el énfasis formal y el acento atomista"(p.::).

De acuerdo con lo anterior se hace imperioso que la formación de docentes de Lengua castellana, en particular, le conceda un lugar preponderante a la pedagogía, toda vez que así se aseguraría una actitud crítica y creativa frente a la necesidad ya planteada. El énfasis en lo pedagógico podría orientarse de acuerdo con los cuatro pilares que plantea Moreno (1998) como características necesarias de un nuevo paradigma pedagógico: productividad, semanticidad, relacionalidad e intersubjetividad.

Por otra parte, es importante tener en cuenta que la formación de docentes ha estado atravesada por una serie de tensiones que afecta de una manera estructural la calidad en dicha formación. La pérdida de prestigio de la profesión docente se adjudica, entre otras causas, al desmejoramiento de las condiciones laborales, en América Latina; al descuido en la calidad de la formación y a que aparecen - especialmente para las mujeres - profesiones que apetecen su talento y que resultan mucho más atractivas, en términos de prestigio y condiciones laborales. En este sentido, Dussel (2005), muestra el desfase en lo que se piensa que es la profesión docente y sus resultados efectivos. Toda esta dinámica real se enfrenta con el propósito de formar docentes idóneos, críticos y creativos. Dinámica que se ha convertido en una preocupación desde siempre en Colombia y que bien retoma Moreno (1998):

El pensamiento oficial acerca del tema no parece ser distinto:

el mismo Ministerio de Educación en el documento citado (Niño Diez, 1998) con el que prologa los nuevos decretos 
reglamentarios de la facultades de educación en el país, es recurrente en situar "la comprensión integral", "la realización de las posibilidades intelectuales", "la preparación de las mentes para la comprensión y construcción de conocimientos", " la capacidad de innovación", "el apoyo al desarrollo de habilidades de pensamiento", como los pilares programáticos de la nueva visión pedagógica".

Para Portilla (2002), el docente debe ser formado, no solo en sus competencias docentes, es decir pedagógicas-didácticas, sino que debe existir la intención explícita de mejorar sus competencias sociales y personales, como una condición crítica para el mejoramiento general de la educación. La calidad de la formación docente debe contar como horizonte transversal con el asunto pedagógico, como eje fundamental de desarrollo. Lizarazo (2006), lo expone de la siguiente manera:

Una pedagogía fantástica buscaría entonces entrenar la facultad creadora, la capacidad de rebasar las rutinas, las organizaciones, los límites, se interesaría en mostrar cada día, a cada paso, la posibilidad de hacer las cosas distintas, de redefinir lo establecido, de repensar lo planteado... Pedagogía fantástica que se plantearía la formación personal y el trayecto social sobre el principio creador, que para Vygotski (1982) se sostiene en dos dimensiones irreductibles de la persona: e I pensamiento y las emociones". $(p,::)$

\section{Hallazgos / discusión:}

Este desplazamiento - que se siente más como un bloqueo - del hacer pedagógico en la formación de nuestros maestros y específicamente de los de lengua castellana, tiene varias causas: la primera es general, se trata de un encantamiento mayúsculo por los saberes con apariencia científica, encantamiento que sin duda es promovido y amplificado por la imagen de minusvalía que, como ya se dijo arriba, proyecta la educación como profesión, lo mismo que por la percepción de que la educación constituye un ejercicio simple, intuitivo, sin fundamento teórico, que de la actividad docente tiene la sociedad en general incluida allí la misma comunidad educativa (Pérez, 2014). 
Desde otra perspectiva, es cierto que la literatura, (el arte que emociona e introduce en mundos nuevos) despierta mucho fervor en los aspirantes a maestros de lengua castellana y es cierto también que ese fervor, generalmente no va más allá del goce inicial y que no se traduce en hecho pedagógico, no se traspone didácticamente, no se convierte en obsesión, ni pretexto formal de enseñanza. Entre otras razones porque se asume - en los maestros y directivos académicos mismos - como ley que el asunto literario, por su esencia estética, es plenamente subjetivo, desconociendo que la escritura, como todo aprendizaje, tiene una técnica y dejando de lado el hecho de que es enseñable (de Almeida, Santos, \& Porto, 2016).

Además, otras de las posibilidades para el ya mencionado desplazamiento de la actividad profesional del docente de lengua castellana, tendrían que ver con que no hay suficiente oferta y en consecuencia, se ven obligados a buscar otras alternativas de trabajo, ya sea porque la oferta que se tiene no es suficientemente atractiva o porque los salarios son muy malos, y las condiciones de trabajo y/o contratación no son las mejores. Esto está conectado con muchos otros aspectos: habría que preguntarse por qué no hay buenas condiciones de trabajo para un área tan importante, si se supone que la enseñanza de la lengua constituye no solamente un área del saber epistemológico que debe tener toda la población, sino que, también, la lengua conforma un recurso estratégico de la identidad.

Lo anteriormente expuesto lleva a un problema más de fondo que tiene que ver con la política, que no está valorando la profesión docente, por lo que probablemente los espacios de trabajo no son de la calidad que este campo amerita.

El otro ámbito, tiene que ver con inclinaciones más de orden individual o de orden personal, lo que lleva a que, muy comúnmente, la gente estudia algo y en el camino descubre que su vocación es otra, que los asuntos que le interesan son de otro tipo (Tahull, 2016).

La última posibilidad - más esperanzadora que las anteriores - se genera en que las competencias y saberes que adquirió el docente de Lengua castellana, en su área de formación, las aplica mejor en otro ámbito. Por ejemplo, casi todos los editores tienen un aprecio especial por la lengua y en ese sentido el destino de una persona que se educó para ser docente de lengua no es demasiado distante si termina trabajando 
como editor o si termina trabajando en el montaje de obras escénicas, de dramaturgia. Entonces ahí habría que conjeturar cómo de alguna manera hay un conjunto de campos, de líneas profesionales o de áreas de trabajo que están más directamente relacionados con la formación disciplinar que con la profesional; insistiendo, eso sí, como se dijo antes en que tanto una como otra área no se están asumiendo con la rigurosidad necesaria.

\section{Conclusiones}

La enseñanza de la lengua castellana no está teniendo la relevancia institucional, social, ni cultural que le corresponde.

Es fundamental revisar la cuestión de la oferta y la demanda de docentes de lengua castellana.

Es claro que hay una dinámica socioinstitucional y cultural en la que hay una especie de subvaloración de la importancia de la lengua, en términos de su formación epistémica y socio cívica, y eso quizás esté mostrando una debilidad en las políticas institucionales o las políticas de gobierno al respecto; es decir, que se hacen necesarias unas políticas de cultura y unas políticas de la educación en las que la lengua ocupe un lugar significativo.

La enseñanza de la lengua materna está vinculada con la educación básica. La educación básica generalmente ha sido un área estratégica de los Estados, pero cada vez con mayor intensidad, participan instituciones privadas y también indirectamente, los medios de comunicación.

Por todo lo anterior, valorizar el ejercicio pedagógico es una tarea que exige que los planes de estudio incluyan con decisión teorías vigentes sobre el niño en todas sus dimensiones humanas, el aprendizaje, la enseñanza, y sobre todo, que dichas teorías se presenten y asuman con criterio aplicado y con la mira siempre puesta en el principio rector de la pedagogía constructiva: Aprender y enseñar son acciones esencialmente creativas, creadoras, sin las cuales las sociedades que llamamos del conocimiento no podrían evolucionar satisfactoriamente.

Esta tarea reclama, asimismo, programas de inducción y sensibilización pedagógica a los maestros que inician su formación. Pero, sobre todo, 
requiere que los líderes políticos de nuestra sociedad acepten y obren en consonancia la idea de que la educación es el centro de operaciones de toda comunidad humana.

\section{Referencias bibliográficas}

Aparicio, O.Y. (2018). Las TIC como herramientas cognitivas. Revista Interamericana de Investigación, Educación y Pedagogía, RIIEP, 11(1). https://orcid.org/0000-0003-35356288

Azevedo, S. R. J. (2014). La composición histórica de la educación religiosa como componente curricular. Revista Interamericana de Investigación, Educación y Pedagogía, RIIEP, 7(1). DOI: https://doi.org/10.15332/s1657-107X.2014.0001.03

Cañón, A. Cedeño M., (2015). Tendencias en la formación de docentes de lengua castellana en América Latina. Informe final de investigación. Bogotá: Universidad Santo Tomás.

de Almeida, R. R., Santos, M. F., \& Porto, J. C. (2016). Lectura de textos ficcionales y el enfoque escolar de literatura: contribuciones para una Pedagogía de la Elección. Revista Interamericana de Educación, Pedagogía y Estudios Culturales, 9(1), 35-51. DOI: https://doi.org/10.22490/25391887.1925

de la Calle, C. V., Malaver, M. O., Gallego, J. D. M., Rodríguez, M., Flórez, J. C., Henao, C. E. \& Saldaña, R. (2014). Aportes de los doctorados de educación en ciencia, tecnología y sociedad, desde la sistematización de sus investigaciones doctorales científicas y formativas, 2000-2010. Revista Interamericana de Investigación, Educación y Pedagogía, RIIEP, 7(1). DOI: https://doi.org/10.15332/s1657-107X.2014.0001.04

Dussel, I Southwell, M (2005). ¿Qué es una buena escuela? Revista El Monitor de la Educación, 5 (5).

Ferreyra, H. A. (2014). Mesas Socioeducativas para la Inclusión y la Igualdad. Un programa "De todos con todos". Una experiencia en construcción. Revista Interamericana de Investigación, Educación y Pedagogía, RIIEP, 7(2). DOI: https://doi. org/10.15332/s1657-107X.2014.0002.01

Konieczny, P. (2015). Lorenzo García Aretio: bases, mediaciones y futuro de la educación a distancia en la sociedad digital. Revista Interamericana de Investigación, Educación y Pedagogía, RIIEP, 8(1). DOI: https://doi.org/10.15332/s1657-

107X.2015.0001.08 
Langer, E. (2016). La construcción de confianza para el estudio de prácticas de resistencia en la escolarización de jóvenes en contextos de pobreza urbana. Revista Interamericana de Investigación, Educación y Pedagogía, RIIEP, 9(2). DOI: https://doi. org/10.22490/25391887.1945

Lizarazo, D. (2006). El espacio lúdico. SEP México, Televisión Educativa, CETE, PITCE: México.

Ministerio de Educación Nacional. Decreto 0272, Febrero 11 de 1998. Bogotá Colombia.

Moreno, J. (1998) "Educar para pensar; pensar para crear; crear para aprender y aprender para crecer". Revista Papeles, Universidad Antonio Nariño, 4 (4).

Pérez, T. H. (2014). Colombia: de la educación en emergencia hacia una educación para el posconflicto y la paz. Revista Interamericana de Investigación, Educación y Pedagogía, RIIEP, 7(2). DOI: https://doi.org/10.15332/s1657-107X.2014.0002.06

Portilla, A. (2002). Tesis Doctoral. Universidad Autónoma de Barcelona. Barcelona

Tahull, J. (2016). Modernidad, educación y género. El proyecto inacabado. Revista Interamericana de Investigación, Educación y Pedagogía, RIIEP, 9(2), 159-178. DOI: https://doi.org/10.22490/25391887.1947

Viejo, C. M., Cabezas, I. L., \& Martínez, M. D. J. I. (2013). Las redes de académicas en la docencia universitaria. Revista Interamericana de Investigación, Educación y Pedagogía, RIIEP, 6(2). DOI: https://doi.org/10.15332/s1657-107X.2013.0002.03 